\title{
Low-dose MDCT: evaluation of the impact of systematic tube current reduction and sparse sampling on the detection of degenerative spine diseases
}

\author{
Nico Sollmann ${ }^{1,2}$ (D) Kai Mei ${ }^{3,4}$. Isabelle Riederer ${ }^{1}$ - Monika Probst ${ }^{1}$ - Maximilian T. Löffler ${ }^{1} \cdot$ Jan S. Kirschke $^{1,2}$. \\ Peter B. Noël ${ }^{3,4}$. Thomas Baum ${ }^{1}$
}

Received: 7 April 2020 / Revised: 29 July 2020 / Accepted: 9 September 2020 / Published online: 18 September 2020

(C) The Author(s) 2020

\begin{abstract}
Objectives To investigate potential radiation dose reduction for multi-detector computed tomography (MDCT) exams of the spine by using sparse sampling and virtually lowered tube currents combined with statistical iterative reconstruction (SIR).

Methods MDCT data of 26 patients $(68.9 \pm 11.7$ years, $42.3 \%$ males) were retrospectively simulated as if the scans were acquired at $50 \%, 10 \%, 5 \%$, and $3 \%$ of the original X-ray tube current or number of projections, using SIR for image reconstructions. Two readers performed qualitative image evaluation considering overall image quality, artifacts, and contrast and determined the number and type of degenerative changes. Scoring was compared between readers and virtual low-dose and sparsesampled MDCT, respectively.

Results Image quality and contrast decreased with virtual lowering of tube current and sparse sampling, but all degenerative changes were correctly detected in MDCT with 50\% of tube current as well as MDCT with 50\% of projections. Sparse-sampled MDCT with only $10 \%$ of initial projections still enabled correct identification of all degenerative changes, in contrast to MDCT with virtual tube current reduction by $90 \%$ where non-calcified disc herniations were frequently missed (R1: $23.1 \%$, R2: $21.2 \%$ non-diagnosed herniations). The average volumetric CT dose index $\left(\mathrm{CTDI}_{\mathrm{vol}}\right)$ was $1.4 \mathrm{mGy}$ for MDCT with $10 \%$ of initial projections, compared with $13.8 \mathrm{mGy}$ for standard-dose imaging.

Conclusions MDCT with 50\% of original tube current or projections using SIR still allowed for accurate diagnosis of degenerative changes. Sparse sampling may be more promising for further radiation dose reductions since no degenerative changes were missed with $10 \%$ of initial projections.

\section{Key Points}

- Most common degenerative changes of the spine can be diagnosed in multi-detector CT with $50 \%$ of tube current or number of projections.

- Sparse-sampled multi-detector CT with only $10 \%$ of initial projections still enables correct identification of degenerative changes, in contrast to imaging with $10 \%$ of original tube current.

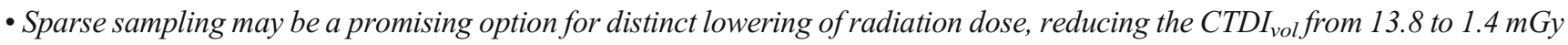
in the study cohort.
\end{abstract}

Keywords Artifacts · Intervertebral disc disease · Intervertebral disc degeneration · Image processing, computer-assisted . Radiation dosage

Nico Sollmann

Nico.Sollmann@tum.de

1 Department of Diagnostic and Interventional Neuroradiology, Klinikum rechts der Isar, Technische Universität München, Ismaninger Str. 22, 81675 Munich, Germany

2 TUM-Neuroimaging Center, Klinikum rechts der Isar, Technische Universität München, Munich, Germany
3 Department of Diagnostic and Interventional Radiology, Klinikum rechts der Isar, Technische Universität München, Ismaninger Str. 22, 81675 Munich, Germany

4 Department of Radiology, Perelman School of Medicine, University of Pennsylvania, 3400 Spruce Street, One Silverstein, Philadelphia PA 19104,, USA 


$\begin{array}{ll}\text { Abbreviations } \\ \text { BMD } & \text { Bone mineral density } \\ \text { CT } & \text { Computed tomography } \\ \text { CTDI } & \text { Volumetric CT dose index } \\ \text { FBP } & \text { Filtered back projection } \\ \text { FE } & \text { Finite element } \\ \text { FOV } & \text { Field of view } \\ \text { ICC } & \text { Intraclass correlation coefficient } \\ \text { IR } & \text { Iterative reconstruction } \\ \text { LD } & \text { Low dose } \\ \text { MDCT } & \text { Multi-detector CT } \\ \text { MRI } & \text { Magnetic resonance imaging } \\ \text { PACS } & \text { Picture archiving and communication system } \\ \text { R1 } & \text { Reader 1 } \\ \text { R2 } & \text { Reader 2 } \\ \text { SD } & \text { Standard dose } \\ \text { SIR } & \text { Statistical IR }\end{array}$

\section{Introduction}

Back pain with or without radiculopathy has high prevalence worldwide and is a major reason for seeking medical advice [1-3]. Magnetic resonance imaging (MRI) is considered the imaging modality of choice for patients with refractory back pain, but computed tomography (CT) still is at the forefront of imaging particularly when rapid MRI is not available or contraindicated. CT can also act complementary to MRI, thanks to its potential to visualize mineralized bone with high contrast, providing additional valuable information to MRI in case of inconclusive findings. CT has proven to be accurate in cases of spinal or neural foraminal stenosis, particularly for distinguishing soft disc herniation from osteophytes, and it can depict bony lysis zones [4-6]. However, diagnostic CT of the spine exposes the patient to a considerable radiation dose, with radiation due to $\mathrm{CT}$ imaging showing drastic rises over the recent years $[7,8]$. Conventional imaging by CT can entail estimated effective doses of approximately $5.6 \mathrm{mSv}$ and $10.0 \mathrm{mSv}$ for the lumbar and whole dorsal spine, respectively $[9,10]$. Such radiation exposure as applied during CT acquisition is correlated with increased estimated cancer risk ratios that need to be considered in the light of patient safety [9-11].

Over the recent decades, several approaches on both the image acquisition side and image reconstruction side have been further developed for CT to lower radiation exposure while keeping image quality sufficient for diagnostic purposes. At the cervical spine, low-dose (LD) multi-detector CT (MDCT) achieved by lowering of tube currents was compared with standard-dose (SD) imaging using filtered back projection (FBP) and hybrid iterative reconstruction (IR), showing that LD imaging with IR provides better image quality for intervertebral discs, neural foramina, and ligaments, and worse image quality for soft tissues and vertebrae when compared with SD imaging with FBP in patients with chronic cervical pain and/or radiculopathy [12]. Another study used LD MDCT with FBP and hybrid IR, showing that high strength levels of IR are favorable for the intervertebral discs and the content of neural foramina at the cervical spine [13]. At the lumbar spine in patients with back pain, LD CT using model-based IR showed better tissue differentiation than hybrid IR or SD CT with FBP [14]. For patients with low to moderate body mass index and chronic back pain, ultra-LD CT protocols with hybrid IR have been tested, indicating preserved image quality and diagnostic accuracy [15]. Furthermore, an earlier study performed systematic simulations of lowered tube currents, indicating that tube charge settings could be reduced to $65 \%$ of SD imaging for diagnostics in patients with suspected lumbar disc herniation [16].

The small body of referenced previous studies on LD CT in patients with back pain with or without radiculopathy achieved reduced radiation exposure by lowering tube currents, partially combined with IR algorithms [12-16]. Modern advances like sparse sampling, a novel technique that is referred to as the acquisition of fewer projection images during scanning, have not been evaluated for diagnostic CT of the degenerative spine. However, sparse sampling reflects a promising alternative since the energy delivery for the individual projection image is maintained while overall radiation exposure is lowered as a consequence of decreased projection numbers $[17,18]$. This can result in preserved image quality while the influence of electronic readout noise on image quality can be circumvented. When combined with advanced image reconstruction, such as statistical IR (SIR), image noise could be further suppressed and radiation doses lowered while structural image information can be preserved [17, 19-23].

Against this background, the present study investigates the potential of sparse-sampled MDCT combined with SIR to reduce radiation exposure for imaging of the degenerative spine. We hypothesized that sparse-sampled MDCT enables greater reductions in radiation dose when compared with MDCT with simulated lowered tube currents while delivering images with sufficient diagnostic quality.

\section{Materials and methods}

\section{Study design and patient inclusion}

This retrospective study was approved by the local institutional review board and was conducted in accordance with the Declaration of Helsinki. Patients who underwent noncontrast MDCT imaging of the spine at our department according to clinical indication (suspected degenerative spine disease or follow-up in degenerative spine disease) during a period of 1 month (June/July 2019) were identified in our hospital's picture archiving and communication system 
(PACS). Exclusion criteria were (1) age below 18 years, (2) motion artifacts in imaging data, (3) previous surgery with instrumentation at the spine, (4) presence of any implants in the field of view (FOV), and (5) vertebral fractures, malignant bone lesions, or spondylodiscitic lesions captured by the FOV. Overall, 26 patients were eligible and included in this study.

\section{Imaging by multi-detector computed tomography}

Image acquisition was performed in supine position using a 128-slice MDCT scanner (Ingenuity Core 128, Philips Healthcare). An initial scout scan was used for planning of the FOV, and subsequent helical scanning was acquired with implicit tube current modulation. Table 1 shows scanning details for MDCT imaging.

\section{Simulations and image reconstruction}

\section{Tube current reduction}

Initial preprocessing of imaging data used a total-variation method for the projection data to reduce image noise $(\lambda=$ $0.01, n=50)[24,25]$. By the use of a simulation algorithm based on raw imaging data, we generated MDCT scans with virtually lowered tube currents in a stepwise fashion [26-31]. The approach for simulations of LD MDCT has been validated previously [31]. Hence, simulations were generated as if MDCT was conducted with 50\% (D50P100), $10 \%$ (D10P100), 5\% (D5P100), and 3\% (D3P100) of the original $\mathrm{X}$-ray tube current. The original imaging data was defined as D100P100.

\section{Sparse sampling}

Sparse sampling was simulated by reading only a reduced amount of projection angles and by deleting the remaining projections in the sinogram [27-29, 32]. The original imaging was defined as D100P100, and virtual sparse-sampled imaging was generated as if MDCT was performed with only $50 \%$ (D100P50), 10\% (D100P10), 5\% (D100P5), and 3\% $(\mathrm{D} 100 \mathrm{P} 3)$ of the original projection data.

\section{Statistical iterative reconstruction}

For image reconstruction of simulated MDCT with lowered tube current or sparse sampling, we used the same in-house developed SIR algorithm that was based on ordered-subset separable paraboloidal surrogate combining a momentum accelerating approach $[33,34]$. A Gaussian noise model was applied and the likelihood term for SIR was computed with log-converted projection data. To enhance convergence and to further depress image noise while achieving adequate bone/ soft tissue contrast, a regularization term based on a Huber penalty was applied. The distinct strength of the regularization term was selected in consensus with three board-certified radiologists. Linear attenuation coefficients of resulting imaging data were translated to Hounsfield units by using air and water information from the MDCT calibration data.

\section{Qualitative image analysis}

Qualitative image evaluation was performed using the PACS viewer (IDS7, Sectra AB). Two radiologists (reader 1 [R1] and reader 2 [R2], 7 years of experience in radiology each) systematically assessed all reconstructed imaging data in all patients (D100P100, D50P100, D10P100, D5P100, D3P100, D100P50, D100P10, D100P5, and D100P3). Evaluations were performed after patient pseudonymization, and the readers had no access to the clinical reports for original imaging and were unaware of the distinct clinical indication that resulted in MDCT imaging. The readers evaluated the SD scan (D100P100) in consensus reading. All other imaging data were assessed separately, with the readers being strictly blinded to the ratings of each other. In detail, the readers performed evaluations of LD scans in the context of eight reading rounds, with an interval of at least 1 week between each round.
Table 1 Scanning details and image reconstruction

\begin{tabular}{|c|c|}
\hline \multicolumn{2}{|c|}{ Scanning details and image reconstruction } \\
\hline Tube voltage (in $\mathrm{kV}$ ) & $123.1 \pm 7.2(120.0-140.0)$ \\
\hline Tube current (in $\mathrm{mA}$ ) & $321.5 \pm 7.5(309.0-334.0)$ \\
\hline Rotation time (in s) & $0.78 \pm 0.163(0.5,0.75$, or 1$)$ \\
\hline Exposure (in mAs) & $194.5 \pm 56.8(130.0-314.0)$ \\
\hline Voxel spacing (in $\mathrm{mm}^{3}$ ) & $0.39 \times 0.39 \times 0.90$ \\
\hline DLP (in $\mathrm{mGy}^{*} \mathrm{~cm}$ ) & $388.9 \pm 179.9(45.5-782.1)$ \\
\hline Field of view (in mm²) & $200 \times 200$ \\
\hline Slice thickness (in mm) & 0.9 \\
\hline Reformations & Sagittal, axial, coronal \\
\hline Windowing & $\begin{array}{l}\text { Individually adjustable — standard setting: window width } 2500 \mathrm{HU} \text {, } \\
\text { window center } 500 \mathrm{HU} \text { (default setting for bone window) }\end{array}$ \\
\hline
\end{tabular}


Within each round, one reconstructed dataset of each patient was evaluated, with the distinct dataset shown per round being subject to randomization. Furthermore, the order of patient cases was also randomized per reading round.

Overall image quality, overall artifacts, and image contrast were evaluated first based on 5-point Likert scales considering the entire FOV (Table 2). Additionally, the readers performed segment-wise evaluation of degenerative changes to determine the presence or absence of such changes (dichotomous evaluation). Furthermore, in case of detected degenerative changes, the readers had to specify them per segment considering spondylosis, pseudospondylolisthesis, spondylolisthesis (with spondylolysis), non-calcified disc herniation, and disc herniation with calcification. In case of presence of more than one of the mentioned degenerative changes, the readers were requested to provide all segment-specific degenerative changes.

\section{Statistical data analysis}

GraphPad Prism (version 6.0; GraphPad Software Inc.) and SPSS (version 25.0; IBM SPSS Statistics for Windows, IBM Corp.) were used for statistical data analyses. The level of statistical significance was set at $p<0.05$.

For patient details, scanning parameters and dose characteristics, and scores assigned by the readers, descriptive statistics were calculated. Furthermore, the number of segments with reported degenerative changes and the absolute frequency of each specific degenerative change was counted. The number of any missed degenerative changes when compared with consensus reading of the SD scans was noted. Analyses were performed separately for the evaluations of R1 and R2 and for all reconstructed image data, respectively.

To compare overall image quality, overall artifacts, and image contrast of MDCT with virtually lowered tube current or sparse sampling against SD scanning, Wilcoxon matchedpairs signed-rank tests were performed (D100P100 vs. D50P100/D10P100/D5P100/D3P100 and D100P50/ D100P10/D100P5/D100P3 for R1 and R2, respectively). Moreover, Wilcoxon matched-pairs signed-rank tests were also conducted between MDCT with virtually lowered tube current or sparse sampling at each level of reduction (D50P100 vs. D100P50, D10P100 vs. D100P10, D5P100 vs. D100P5, and D3P100 vs. D100P3 for R1 and R2, respectively). Intraclass correlation coefficients (ICCs) were computed to assess inter-reader agreement (two-way mixed model).

\section{Results}

\section{Cohort characteristics}

Image data of 26 patients (mean age: $68.9 \pm 11.7$ years, range: $37.5-86.9$ years, $42.3 \%$ males) were used in this study, with simulations of sparse-sampled MDCT or MDCT with lowered tube current being available from all patients. Scans covered the cervical spine (in $23.1 \%$ of patients) or lumbosacral spine (in $76.9 \%$ of patients), with a median of 6.5 segments being included in the FOV (range: 3-10 segments) (Figs. 1, 2, 3, 4, and 5).

In total, degenerative spine disease affected 84 segments when considering the whole cohort. Spondylosis was most common (68 incidences, $50 \%$ of detected degenerative changes), followed by non-calcified disc herniation ( 52 incidences, $38.2 \%$ of detected degenerative changes) and pseudospondylolisthesis (8 incidences, $5.8 \%$ of detected degenerative changes, $100 \%$ Meyerding grade I) as well as spondylolisthesis (with spondylolysis; 4 incidences, 3.0\% of detected degenerative changes, 50\% Meyerding grade I and $50 \%$ Meyerding grade II). Calcified disc herniation (4 incidences, $3.0 \%$ of detected degenerative changes) occurred infrequently.

\section{Qualitative image analysis}

Compared with SD scanning, both virtual tube current reduction and sparse sampling decreased overall image quality and image contrast and increased overall artifacts, with significant differences $(p<0.05)$ between SD MDCT and all levels of virtually lowered tube current or sparse sampling except for D100P50 (overall image quality: R2: $p=0.25$; overall

Table 2 Scoring scheme for qualitative image analysis

Qualitative image analysis

\begin{tabular}{llllll}
\hline Item & Score & & & \\
\cline { 2 - 6 } & 1 & 2 & 3 & 5 \\
\hline Overall image quality & Very good to perfect quality & Good to very good quality & Medium quality & Poor quality & Inappropriate quality \\
Overall artifacts & No artifacts & Minimal artifacts & Prominent artifacts & Major artifacts & Severe artifacts \\
Image contrast & Very good to perfect contrast & Good to very good contrast & Medium contrast & Poor contrast & Inappropriate contrast \\
\hline
\end{tabular}




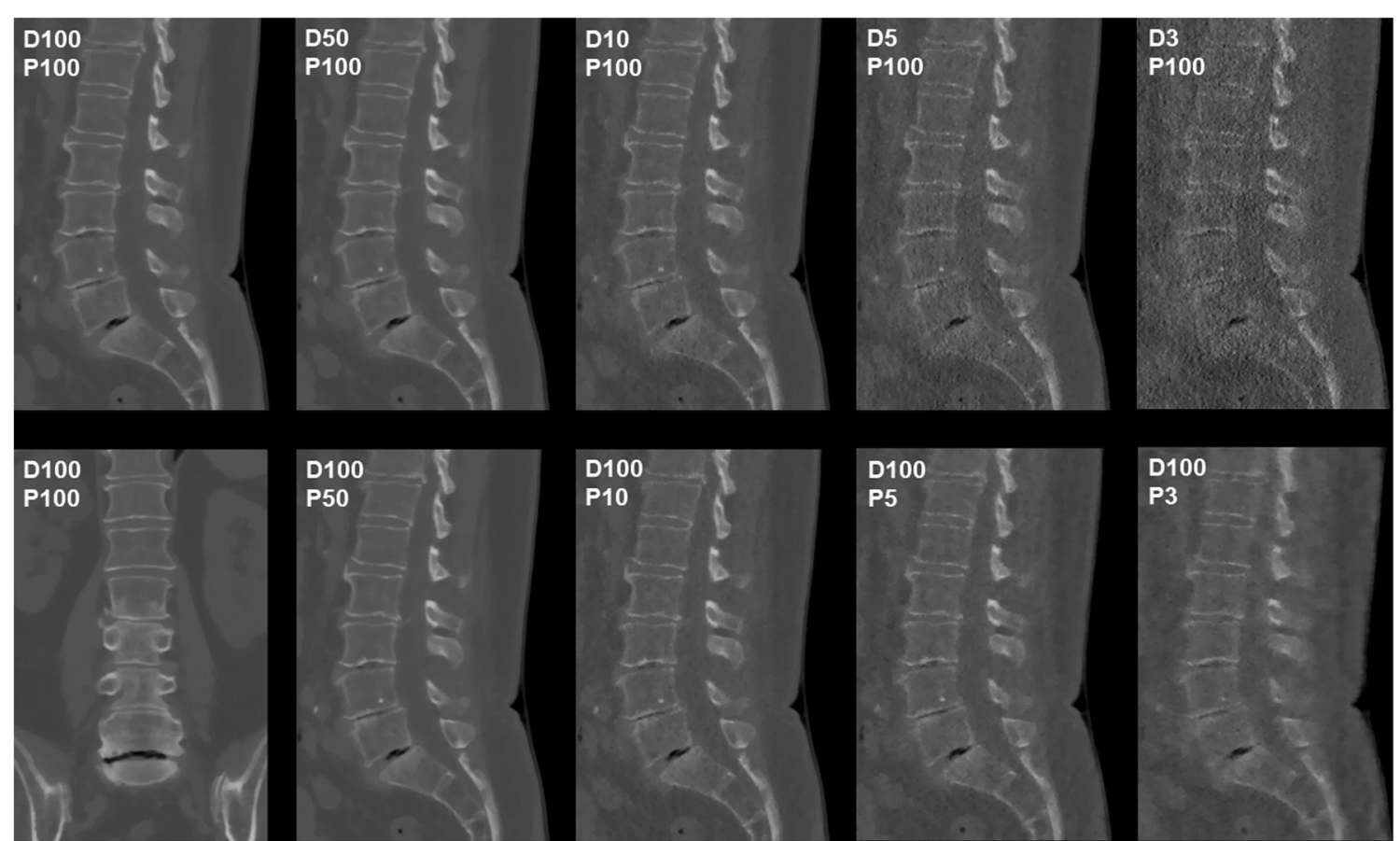

Fig. 1 Patient case 1-lumbar degenerative changes (bone window). This figure depicts the lumbosacral spine of a 61-year-old female patient in standard-dose (SD) scans (D100P100, sagittal and coronal plane, bone window) and in simulated scans with virtual reduction of tube current (D50P100, D10P100, D5P100, and D3P100, sagittal plane, bone window) or sparse sampling (D100P50, D100P10, D100P5, and $\mathrm{D} 100 \mathrm{P} 3$, sagittal plane, bone window). For image evaluation, all planes were available to the readers with individually adaptable windowing options. The patient showed multi-segmental non-calcified disc herniations and spondylosis according to original imaging

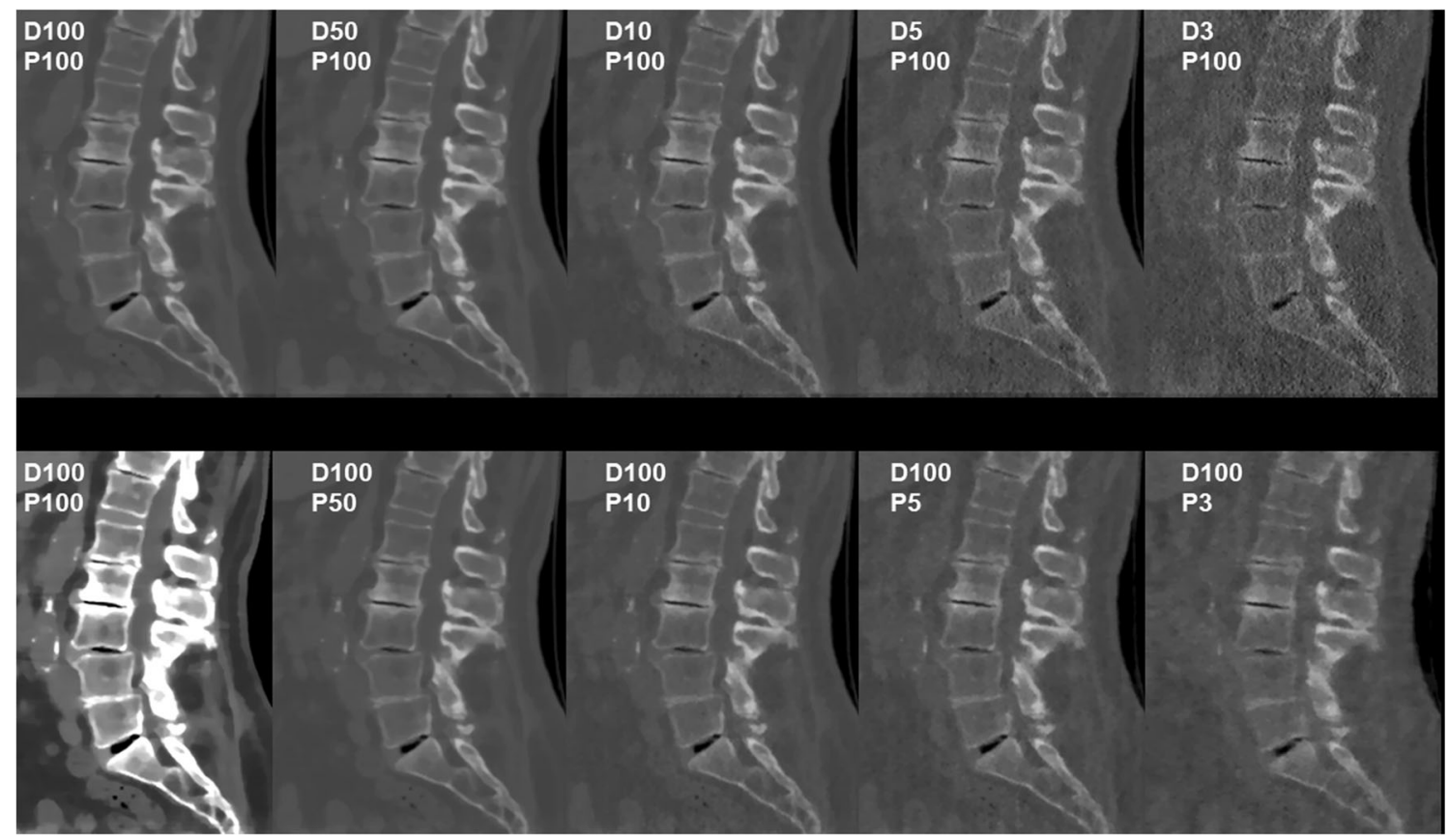

Fig. 2 Patient case 2-lumbar degenerative changes (bone window). This figure shows the lumbosacral spine of an 82-year-old female patient in standard-dose (SD) scans (D100P100, sagittal plane, bone and soft tissue window) and in simulated scans with virtual reduction of tube current (D50P100, D10P100, D5P100, and D3P100, sagittal plane, bone window) or sparse sampling (D100P50, D100P10, D100P5, and
D100P3, sagittal plane, bone window). For image evaluation, all planes were available to the readers with individually adaptable windowing options. The patient showed multi-segmental non-calcified disc herniations, spondylosis, and pseudospondylolisthesis (L3/4) according to original imaging 


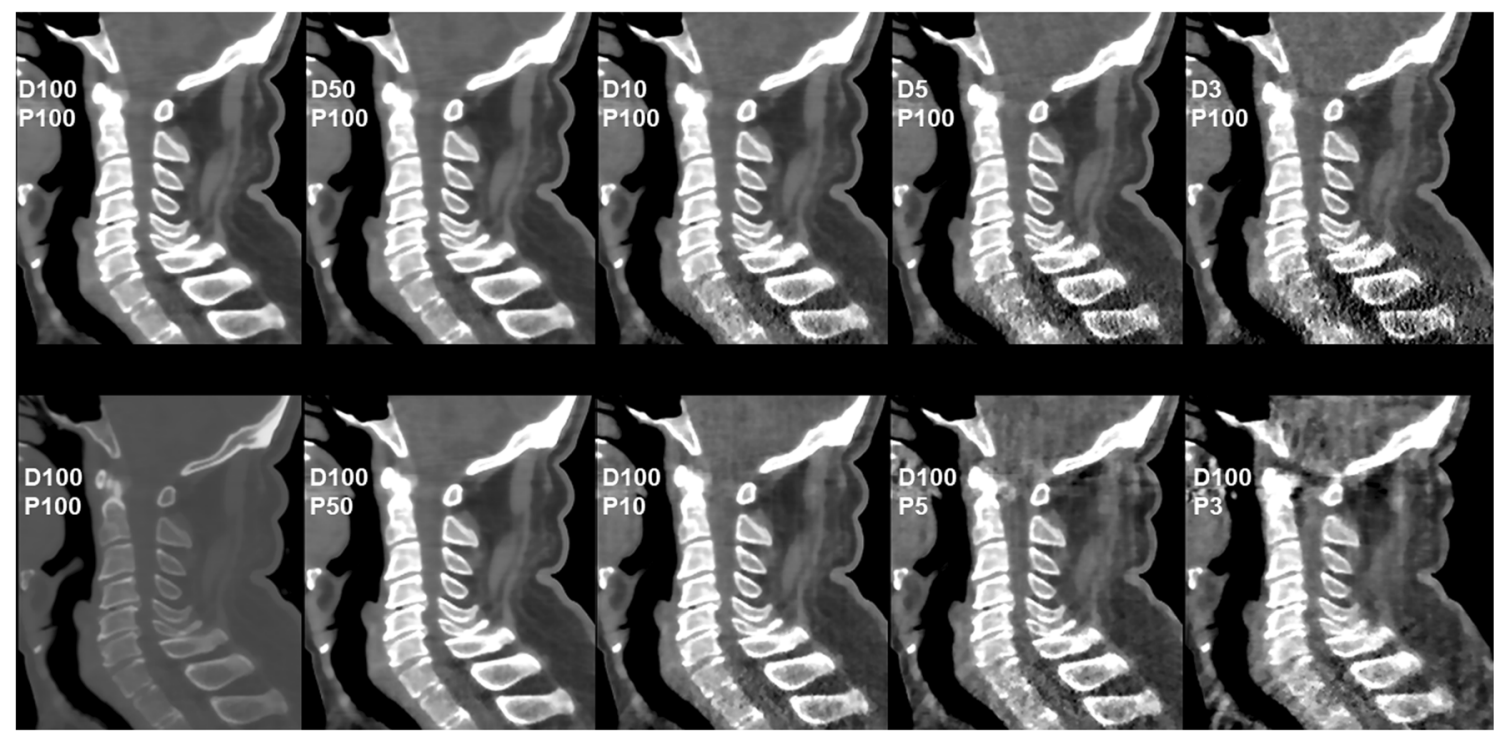

Fig. 3 Patient case 3 - cervical degenerative changes (soft tissue window). This figure depicts the cervical spine of a 63-year-old male patient in standard-dose (SD) scans (D100P100, sagittal plane, bone and soft tissue window) and in simulated scans with virtual reduction of tube current (D50P100, D10P100, D5P100, and D3P100, sagittal plane, soft

artifacts: R2: $p=0.50$; image contrast: $\mathrm{R} 1: p=0.06, \mathrm{R} 2$ : $p=0.13$ ).

However, sparse-sampled MDCT at 50\%, 10\%, and 5\% of original projections showed better results when compared with MDCT with virtual reduction of tube current by $50 \%$, tissue window) or sparse sampling (D100P50, D100P10, D100P5, and D100P3, sagittal plane, soft tissue window). For image evaluation, all planes were available to the readers with individually adaptable windowing options. The patient showed multi-segmental non-calcified disc herniations and spondylosis according to original imaging

$10 \%$, and $5 \%$ (Table 3). Specifically, imaging data of D100P10 still showed good to very good image quality and contrast with only minimal artifacts (Table 3). Agreement between readers was fair to excellent for overall image quality (ICC range: $0.40-0.91$ ), good to excellent for overall artifacts

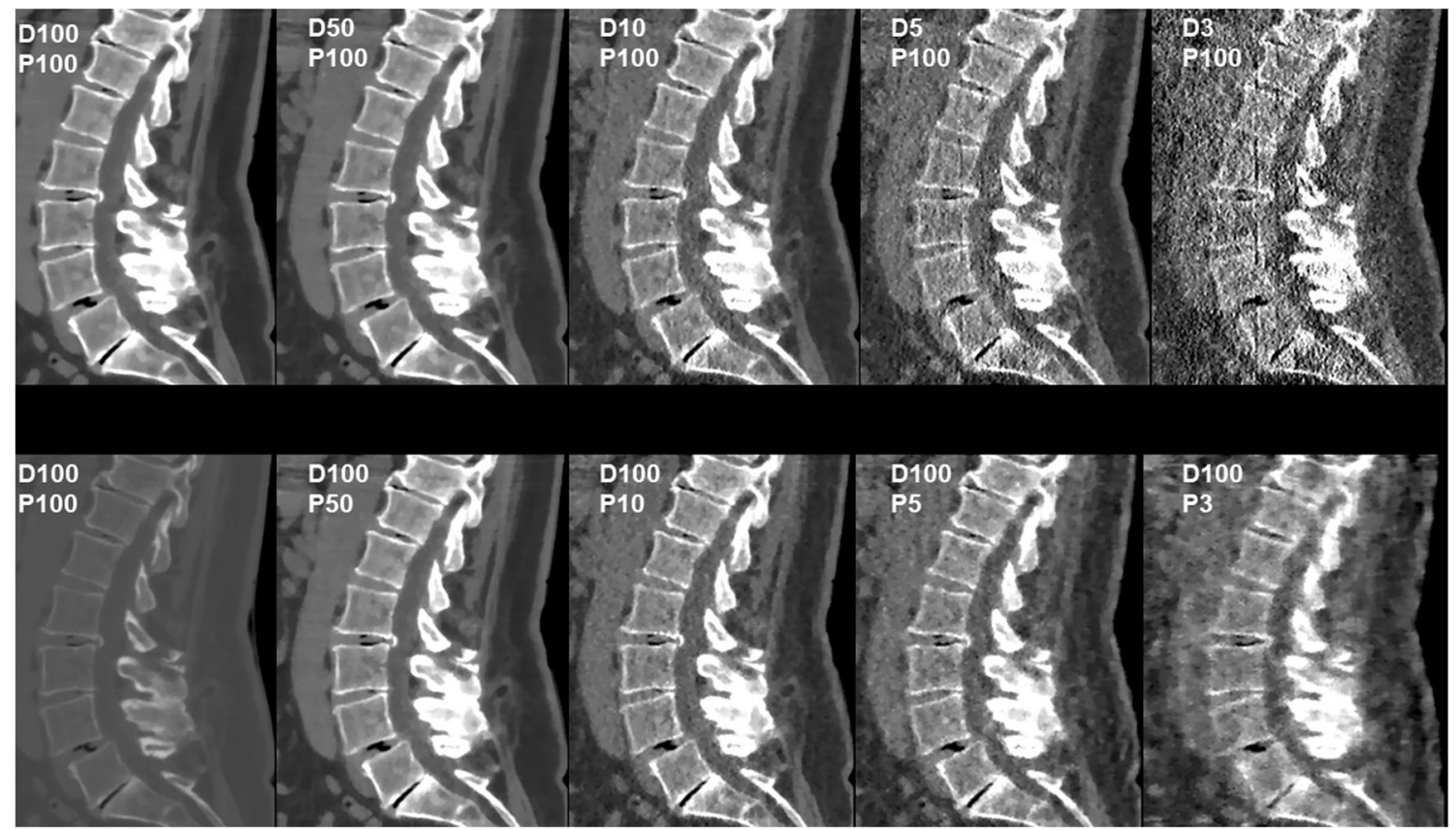

Fig. 4 Patient case 4-lumbar degenerative changes (soft tissue window). This figure shows the lumbar spine of a 66-year-old female patient in standard-dose (SD) scans (D100P100, sagittal plane, bone and soft tissue window) and in simulated scans with virtual reduction of tube current (D50P100, D10P100, D5P100, and D3P100, sagittal plane, soft tissue window) or sparse sampling (D100P50, D100P10, D100P5, and
D100P3, sagittal plane, soft tissue window). For image evaluation, all planes were available to the readers with individually adaptable windowing options. The patient showed multi-segmental disc herniations (with calcified components L 2/3), spondylosis, and pseudospondylolisthesis (L4/5) according to original imaging 
Fig. 5 Patient case 5-lumbar degenerative changes (soft tissue window). This figure depicts the lower thoracic to lumbosacral spine of a 59-year-old male patient in standard-dose (SD) scans (D100P100, sagittal plane, bone and soft tissue window) and in simulated scans with virtual reduction of tube current (D50P100, D10P100, D5P100, and D3P100, sagittal plane, soft tissue window) or sparse sampling (D100P50, D100P10, D100P5, and D100P3, sagittal plane, soft tissue window). For image evaluation, all planes were available to the readers with individually adaptable windowing options. The patient showed multi-segmental non-calcified disc herniations and spondylosis according to original imaging

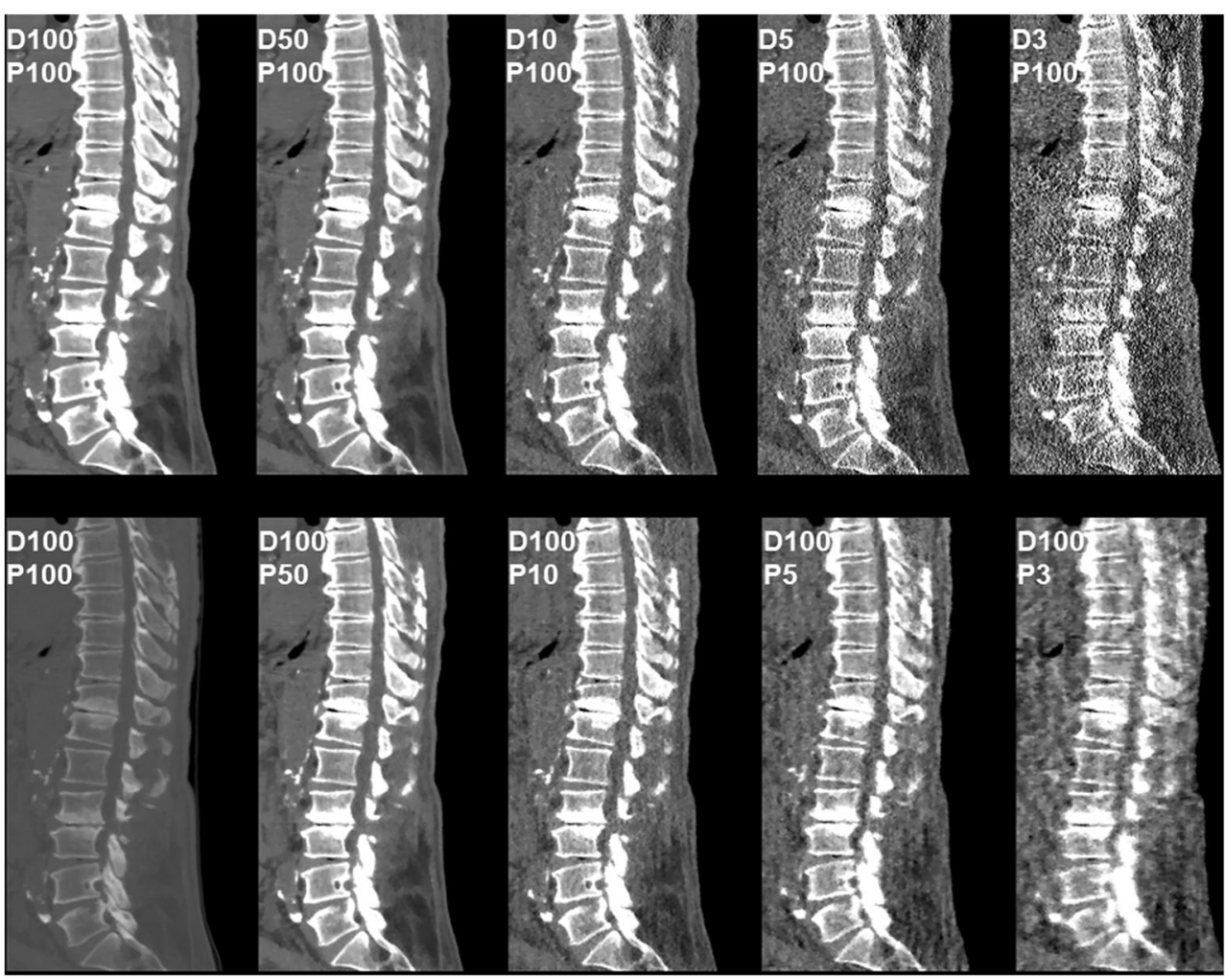

(ICC range: 0.65-0.92), and good to excellent for image contrast (ICC range: 0.84-0.99; Table 3).

A $50 \%$ reduction of tube current or projection numbers still allowed for correct detection of all degenerative changes (Table 4). Furthermore, using sparse-sampled MDCT with
$10 \%$ of original projection numbers still allowed for detection of all segments affected by degenerative spine disease, in contrast to MDCT with virtual tube currents of $10 \%$ of original imaging (Table 4). All specific degenerative changes were detected by both readers for D100P10, whereas readers

Table 3 Results of qualitative image evaluation

\begin{tabular}{|c|c|c|c|c|c|c|c|c|c|c|c|c|c|}
\hline & D100P100 & D50P100 & D100P50 & $p$ & D10P100 & D100P10 & $p$ & D5P100 & D100P5 & $p$ & D3P100 & D100P3 & $p$ \\
\hline \multicolumn{14}{|c|}{ Overall image quality } \\
\hline $\mathrm{R} 1$ & 1.0 & $1.4 \pm 0.5$ & $1.3 \pm 0.5$ & n.s. & $2.7 \pm 0.6$ & $2.5 \pm 0.5$ & n.s. & $3.7 \pm 0.8$ & $3.5 \pm 0.6$ & n.s. & $4.5 \pm 0.7$ & $4.7 \pm 0.6$ & n.s. \\
\hline $\mathrm{R} 2$ & & $1.3 \pm 0.5$ & $1.1 \pm 0.3$ & n.s. & $2.5 \pm 0.6$ & $2.2 \pm 0.4$ & 0.039 & $3.5 \pm 0.5$ & $3.2 \pm 0.4$ & 0.022 & $4.4 \pm 0.6$ & $4.4 \pm 0.5$ & n.s. \\
\hline ICC & - & 0.83 & 0.47 & - & 0.86 & 0.40 & - & 0.82 & 0.66 & - & 0.91 & 0.66 & - \\
\hline \multicolumn{14}{|c|}{ Overall artifacts } \\
\hline $\mathrm{R} 1$ & 1.0 & $1.4 \pm 0.5$ & $1.2 \pm 0.4$ & n.s. & $2.7 \pm 0.6$ & $2.5 \pm 0.5$ & n.s. & $3.7 \pm 0.7$ & $3.5 \pm 0.6$ & n.s. & $4.5 \pm 0.7$ & $4.7 \pm 0.6$ & n.s. \\
\hline $\mathrm{R} 2$ & & $1.4 \pm 0.5$ & $1.1 \pm 0.3$ & 0.016 & $2.5 \pm 0.5$ & $2.2 \pm 0.4$ & 0.016 & $3.5 \pm 0.5$ & $3.4 \pm 0.5$ & n.s. & $4.5 \pm 0.5$ & $4.5 \pm 0.5$ & n.s. \\
\hline ICC & - & 0.92 & 0.65 & - & 0.80 & 0.68 & - & 0.86 & 0.81 & - & 0.90 & 0.77 & - \\
\hline \multicolumn{14}{|c|}{ Image contrast } \\
\hline R1 & 1.0 & $1.5 \pm 0.5$ & $1.2 \pm 0.4$ & n.s. & $2.7 \pm 0.7$ & $2.5 \pm 0.5$ & n.s. & $3.6 \pm 0.8$ & $3.5 \pm 0.6$ & n.s. & $4.4 \pm 0.9$ & $4.7 \pm 0.6$ & n.s. \\
\hline R2 & & $1.6 \pm 0.5$ & $1.2 \pm 0.4$ & 0.003 & $2.7 \pm 0.7$ & $2.3 \pm 0.5$ & 0.037 & $3.7 \pm 0.7$ & $3.4 \pm 0.6$ & n.s. & $4.4 \pm 0.9$ & $4.6 \pm 0.6$ & n.s. \\
\hline ICC & - & 0.88 & 0.93 & - & 0.99 & 0.84 & - & 0.93 & 0.97 & - & 0.99 & 0.97 & - \\
\hline
\end{tabular}

This table shows the results of qualitative image evaluation by reader 1 (R1) and reader 2 (R2) for overall image quality, overall artifacts, and image contrast (based on 5-point Likert scales). The standard-dose (SD) scans (D100P100) were evaluated in consensus reading; scans with virtual reduction of tube current (D50P100, D10P100, D5P100, and D3P100) or sparse sampling (D100P50, D100P10, D100P5, and D100P3) were evaluated independently. Statistical iterative reconstruction (SIR) was used in all imaging data. Scores as mean \pm standard deviation are depicted; $p$ values indicate statistical significance (values $<0.05$ are given in italics; n.s. not statistically significant) as based on comparisons between imaging with virtually lowered tube current or sparse sampling at each level of reduction (D50P100 vs. D100P50, D10P100 vs. D100P10, D5P100 vs. D100P5, and D3P100 vs. D100P3 for R1 and R2, respectively). Intraclass correlation coefficients (ICCs) are given for inter-reader agreement 
Table 4 Segments and characteristics of degenerative changes

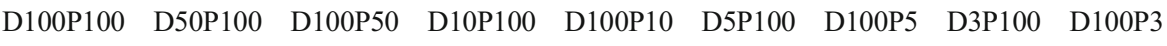

Segments with detected degenerative changes

\begin{tabular}{|c|c|c|c|c|c|c|c|c|c|c|}
\hline \multirow[t]{2}{*}{ Number of segments } & $\mathrm{R} 1$ & 84 & 84 & 84 & $82(2)$ & 84 & $76(8)$ & $78(6)$ & $71(13)$ & $73(11)$ \\
\hline & $\mathrm{R} 2$ & & 84 & 84 & $82(2)$ & 84 & $75(9)$ & $79(5)$ & $72(12)$ & $72(12)$ \\
\hline \multicolumn{11}{|l|}{ Specifics of degenerative changes } \\
\hline \multirow[t]{2}{*}{ Spondylosis } & $\mathrm{R} 1$ & 68 & 68 & 68 & 68 & 68 & 68 & 68 & 68 & 68 \\
\hline & $\mathrm{R} 2$ & & 68 & 68 & 68 & 68 & 68 & 68 & $66(2)$ & 68 \\
\hline \multirow[t]{2}{*}{ Pseudospondylolisthesis } & $\mathrm{R} 1$ & 8 & 8 & 8 & 8 & 8 & 8 & 8 & $5(3)$ & $5(3)$ \\
\hline & $\mathrm{R} 2$ & & 8 & 8 & 8 & 8 & 8 & 8 & $5(3)$ & $5(3)$ \\
\hline \multirow[t]{2}{*}{ Spondylolisthesis (with spondylolysis) } & $\mathrm{R} 1$ & 4 & 4 & 4 & 4 & 4 & 4 & 4 & 4 & 4 \\
\hline & $\mathrm{R} 2$ & & 4 & 4 & 4 & 4 & 4 & 4 & 4 & 4 \\
\hline \multirow[t]{2}{*}{ Non-calcified disc herniation } & $\mathrm{R} 1$ & 52 & 52 & 52 & $40(12)$ & 52 & $14(38)$ & $36(16)$ & $1(51)$ & $4(48)$ \\
\hline & $\mathrm{R} 2$ & & 52 & 52 & $41(11)$ & 52 & $15(37)$ & $27(25)$ & $2(50)$ & $9(43)$ \\
\hline \multirow[t]{2}{*}{ Calcified disc herniation } & $\mathrm{R} 1$ & 4 & 4 & 4 & 4 & 4 & 4 & 4 & 4 & 4 \\
\hline & $\mathrm{R} 2$ & & 4 & 4 & 4 & 4 & 4 & 4 & 4 & 4 \\
\hline
\end{tabular}

This table shows the number of segments with detected degenerative changes and the number of specific changes for the whole cohort according to reader 1 (R1) and reader 2 (R2). The standard-dose (SD) scans (D100P100) were evaluated in consensus reading; scans with virtual reduction of tube current (D50P100, D10P100, D5P100, and D3P100) or sparse sampling (D100P50, D100P10, D100P5, and D100P3) were evaluated independently. Italic numbers in brackets represent the number of missed degenerative changes when compared with SD imaging

missed a considerable fraction of herniated discs in MDCT with virtual reduction of tube current by $90 \%$ (D10P100: non-calcified disc herniation: R1: 12 out of 52 missed, R2: 11 out of 52 missed; Table 4).

\section{Radiation dose}

The mean volumetric $\mathrm{CT}$ dose index $\left(\mathrm{CTDI}_{\mathrm{vol}}\right)$ for SD scanning was $13.8 \pm 5.0 \mathrm{mGy}$ (range: $8.6-27.4 \mathrm{mGy}$ ), and it amounted to $6.9 \mathrm{mGy}$ (D50P100 or D100P50), $1.4 \mathrm{mGy}$ (D10P100 or D100P10), 0.7 mGy (D5P100 or D100P5), and $0.4 \mathrm{mGy}$ (D3P100 or D100P3) for simulated MDCT with tube current reduction or sparse sampling on average, respectively.

\section{Discussion}

This study applied stepwise simulations of lowered tube currents and sparse sampling combined with SIR for MDCT in patients with suspected or previously diagnosed degenerative spine disease. Both virtual lowering of tube currents as well as sparse sampling with SIR were feasible and enabled decreases in radiation doses. However, sparse sampling allowed for greater reductions in radiation exposure when compared to virtual tube current reduction, with MDCT with $10 \%$ of original projections still providing scans with sufficient image quality and diagnostic value since no degenerative changes were missed when compared with original imaging.
Previous literature on LD CT in patients with back pain with or without radiculopathy used lowering of tube currents for dose reductions [12-16]. These studies mostly compared patients who underwent different imaging protocols for $\mathrm{CT}$ of the cervical or lumbar spine $[12,14,15]$. Such studies were successful in reducing radiation exposure, but their study design by comparing mainly two groups of different patients for only two dose levels did not allow to systematically reveal a certain tube current threshold for diagnostic purpose, as this study can provide by using stepwise simulations on an intrasubject level. To the authors' knowledge, only one previous study used a comparable approach (restrictions to 65\%,50\%, $35 \%$, and $20 \%$ of SD imaging), but only focused on disc herniations as one manifestation of spine degeneration and did not use advanced image reconstructions, most probably due to the non-availability at the time of publication [16]. For patients with suspected lumbar disc herniation, tube charge settings could be reduced to $65 \%$ of SD imaging in this previous study [16]. In contrast, results of the present study indicate that more dedicated tube current reductions by $50 \%$ might be possible without relevant restrictions for diagnostic purpose, evaluating a broader range of common degenerative changes at the spine. This may be due to the interim developments of MDCT technology including advancements in image reconstruction, such as SIR.

Sparse sampling is a novel approach that has potential to lower radiation exposure to the patient. It describes the reduction in projection images for scanning with the anticipated benefit of lowered overall energy $[17,18]$. At the spine, previous research demonstrated that determination of bone 
mineral density (BMD) and microstructure of vertebrae using sparse-sampled MDCT is more robust compared with that using MDCT with lowered tube currents [27]. Additionally, images with up to $50 \%$ reductions in radiation dose through sparse sampling can be used for finite element (FE)-based predictions of femoral failure load [35]. Sufficient image quality and diagnostic accuracy for detection of vertebral fractures could be achieved with $50 \%$ of original projections, while, on the contrary, MDCT with 50\% lowered tube currents yielded inferior results [28].

Concerning the degenerative spine, the present study suggests that both sparse sampling and virtual reductions of tube current have potential for dose reduction. In particular, sparse-sampled MDCT with only $10 \%$ of original projections (reduction of CTDI ${ }_{\mathrm{vol}}$ from 13.8 to $1.4 \mathrm{mGy}$ ) generates scans entailing high diagnostic accuracy at sufficient image quality for diagnostic purposes. Thus, when compared with MDCT with virtually lowered tube current, sparse sampling could enable even greater reductions in radiation exposure while both readers were able to identify all degenerative changes without any missed pathology when compared with SD MDCT. This seems to be primarily due to better detectability of non-calcified disc herniations in sparse-sampled MDCT when compared with MDCT with virtual tube current reduction. In this regard, particularly soft tissue contrast seems to be affected in LD scans, with image noise showing considerable increases particularly for virtually reduced tube current, thus hampering unequivocal identification of non-calcified disc herniations. For degenerative changes directly affecting the vertebrae like spondy losis, pseudospondylolisthesis or spondylolisthesis (with spondylolysis), as well as calcified disc herniations, virtual tube current reduction was not clearly inferior to sparse sampling. However, pseudospondylolisthesis was missed in scans simulated with only $3 \%$ of initial tube current or projection numbers, most likely due to fading of bony contours of the vertebrae and related issues with detectability of vertebral shift in the anterior-posterior direction. Of note, all patients with pseudospondylolisthesis of this study only showed a minor degree of listhesis (Meyerding grade I), probably making clear detection of slight vertebral shift impossible for MDCT with very low doses.

The reason for the overall better results of sparse-sampled data most likely relates to the inherently different methods of sparse sampling and tube current reduction. Specifically, while the approach of tube current reduction leads to considerable increases in image noise that can negatively affect diagnostic use, sparse sampling implies maintained energy delivery for the individual projection image, but lowered overall radiation exposure due to the decrease in total projection numbers $[17,18]$. Consequently, preserved image quality for the individual projection can be achieved while circumventing the influence of electronic readout noise, leading to largely preserved structural image information [17, 18].

Sparse sampling reduces the quantity of measurement during the MDCT exam, which can be compensated by advanced reconstruction algorithms. We used in-house-developed SIR for MDCT with virtually lowered tube currents and sparse sampling, respectively. Advanced reconstruction methods such as IR have potential to further suppress noise in imaging data, which increases image quality and can therefore allow for further restrictions in radiation exposure from scanning without relevant loss of structural image information [17, 19-23]. A study investigating the feasibility of SIR in predicting MDCT-based BMD and vertebral bone strength from $\mathrm{FE}$ analysis in comparison to data reconstructed with FBP revealed that SIR produced images of the best quality with regard to noise, signal-to-noise, and contrast-to-noise ratios [36]. Other previous research showed that IR at certain levels can improve image quality of LD CT of the lumbar spine when compared with FBP [37]. Similarly, LD CT of the cervical spine using IR was evaluated, recommending that rather high strength levels of IR might be used for visualization of intervertebral discs and the content of neural foramina [13]. In accordance with these findings, the present study also used a high level of regularization as this was considered superior for evaluations of the degenerative spine following consensus evaluation of different regularization levels. However, most previous work applied a hybrid IR algorithm $[12,13,15]$. Hybrid IR algorithms are generally characterized by reasonable reconstruction speed but are less effective in artifact and noise reduction, with more advanced approaches, such as model-based IR or SIR, facilitating further improvement in image quality [17, 19, 20].

This study also has limitations that should be considered. First, this study only investigated degenerative changes, thus not including pathologies with osseodestructive behavior like spondylodiscitis or bone metastases. Such pathologies can share similar symptoms with degenerative spine diseases and may represent important differential diagnoses. Thus, studies investigating LD MDCT by means of virtual tube current reduction and, importantly, sparse sampling are needed to expand clinical applicability of our approach. Second, LD MDCT was only simulated in this study, which was based on raw data taken from SD MDCT. This is related to the circumstance that MDCT systems capable of employing sparse sampling have not yet been made commercially available, hampering our approach to be seamlessly introduced into the clinical routine. Sparse sampling requires additional controlling units in the X-ray tube, increasing stability demands, and may increase the total cost of the system. Leading MDCT manufacturers have not yet shown sufficient interest in further systematic investigation and extra implementation of the technique, although first prototypes of systems have been developed successfully [38, 39]. Yet, distribution of sparse-sampled 
imaging beyond mere application in the research setting may become possible soon.

In conclusion, tube current reduction and sparse sampling combined with SIR enable decreases in radiation exposure for MDCT of the degenerative spine. However, sparse-sampled MDCT with SIR could lead to greater reductions in radiation exposure, with a $90 \%$ decrease in projection numbers (reduction of $\mathrm{CTDI}_{\mathrm{vol}}$ from 13.8 to $1.4 \mathrm{mGy}$ ) when compared with original imaging still providing sufficient image quality and high diagnostic value since no degenerative changes were missed in this study. Thus, particularly sparse sampling combined with advanced image reconstruction might be a promising option for LD MDCT in patients with suspected degenerative spine disease.

Funding Open Access funding enabled and organized by Projekt DEAL. We acknowledge support through the University of Pennsylvania Research Foundation (URF, to P.B.N.), the Deutsche Forschungsgemeinschaft (DFG, German Research Foundation) Project 432290010 (to J.S.K., T.B, and P.B.N.), the B. Braun Foundation - Project BBST-D-19-00106 (to N.S.), and Philips Healthcare.

\section{Compliance with ethical standards}

Guarantor The scientific guarantor of this publication is Thomas Baum, MD.

Conflict of interest The authors of this manuscript declare no relationships with any companies whose products or services may be related to the subject matter of the article.

Statistics and biometry One of the authors has significant statistical expertise.

Informed consent Written informed consent was not required for this study because of its retrospective character and the analyses being based only on data acquired for clinical routine.

Ethical approval Institutional Review Board approval was obtained.

\section{Methodology \\ - Retrospective \\ - Diagnostic or prognostic study \\ - Performed at one institution}

Open Access This article is licensed under a Creative Commons Attribution 4.0 International License, which permits use, sharing, adaptation, distribution and reproduction in any medium or format, as long as you give appropriate credit to the original author(s) and the source, provide a link to the Creative Commons licence, and indicate if changes were made. The images or other third party material in this article are included in the article's Creative Commons licence, unless indicated otherwise in a credit line to the material. If material is not included in the article's Creative Commons licence and your intended use is not permitted by statutory regulation or exceeds the permitted use, you will need to obtain permission directly from the copyright holder. To view a copy of this licence, visit http://creativecommons.org/licenses/by/4.0/.

\section{References}

1. Freburger JK, Holmes GM, Agans RP et al (2009) The rising prevalence of chronic low back pain. Arch Intern Med 169:251-258

2. Walker BF (2000) The prevalence of low back pain: a systematic review of the literature from 1966 to 1998. J Spinal Disord 13:205217

3. Bouhassira D, Lanteri-Minet M, Attal N, Laurent B, Touboul C (2008) Prevalence of chronic pain with neuropathic characteristics in the general population. Pain 136:380-387

4. Freund M, Sartor K (2006) Degenerative spine disorders in the context of clinical findings. Eur J Radiol 58:15-26

5. Tins B (2010) Technical aspects of CT imaging of the spine. Insights Imaging 1:349-359

6. Parizel PM, Van Hoyweghen AJ, Bali A, Van Goethem J, Van Den Hauwe L (2016) The degenerative spine: pattern recognition and guidelines to image interpretation. Handb Clin Neurol 136:787808

7. Brenner DJ, Hall EJ (2007) Computed tomography-an increasing source of radiation exposure. N Engl J Med 357:2277-2284

8. Fazel R, Krumholz HM, Wang Y et al (2009) Exposure to low-dose ionizing radiation from medical imaging procedures. $\mathrm{N}$ Engl $\mathrm{J}$ Med 361:849-857

9. Richards PJ, George J, Metelko M, Brown M (2010) Spine computed tomography doses and cancer induction. Spine (Phila Pa 1976) 35:430-433

10. Richards PJ, George J (2010) Diagnostic CT radiation and cancer induction. Skeletal Radiol 39:421-424

11. Smith-Bindman R, Lipson J, Marcus R et al (2009) Radiation dose associated with common computed tomography examinations and the associated lifetime attributable risk of cancer. Arch Intern Med 169:2078-2086

12. Becce F, Ben Salah Y, Verdun FR et al (2013) Computed tomography of the cervical spine: comparison of image quality between a standard-dose and a low-dose protocol using filtered backprojection and iterative reconstruction. Skeletal Radiol 42:937-945

13. Omoumi P, Verdun FR, Ben Salah Y et al (2014) Low-dose multidetector computed tomography of the cervical spine: optimization of iterative reconstruction strength levels. Acta Radiol 55:335-344

14. Yang CH, Wu TH, Lin CJ et al (2016) Knowledge-based iterative model reconstruction technique in computed tomography of lumbar spine lowers radiation dose and improves tissue differentiation for patients with lower back pain. Eur J Radiol 85:1757-1764

15. Lee SH, Yun SJ, Jo HH, Kim DH, Song JG, Park YS (2018) Diagnostic accuracy of low-dose versus ultra-low-dose CT for lumbar disc disease and facet joint osteoarthritis in patients with low back pain with MRI correlation. Skeletal Radiol 47:491-504

16. Bohy P, de Maertelaer V, Roquigny A, Keyzer C, Tack D, Gevenois PA (2007) Multidetector CT in patients suspected of having lumbar disk herniation: comparison of standard-dose and simulated low-dose techniques. Radiology 244:524-531

17. Willemink MJ, Noel PB (2019) The evolution of image reconstruction for CT-from filtered back projection to artificial intelligence. Eur Radiol 29:2185-2195

18. Abbas S, Lee T, Shin S, Lee R, Cho S (2013) Effects of sparse sampling schemes on image quality in low-dose CT. Med Phys 40: 111915

19. Willemink MJ, de Jong PA, Leiner T et al (2013) Iterative reconstruction techniques for computed tomography part 1: technical principles. Eur Radiol 23:1623-1631

20. Willemink MJ, Leiner T, de Jong PA et al (2013) Iterative reconstruction techniques for computed tomography part 2: initial results in dose reduction and image quality. Eur Radiol 23:1632-1642 
21. Noel PB, Renger B, Fiebich M et al (2013) Does iterative reconstruction lower CT radiation dose: evaluation of 15,000 examinations. PLoS One 8:e81141

22. Loffler MT, Sollmann N, Mei K et al (2019) X-ray-based quantitative osteoporosis imaging at the spine. Osteoporos Int. https://doi. org/10.1007/s00198-019-05212-2

23. Iyama Y, Nakaura T, Iyama A et al (2017) Feasibility of iterative model reconstruction for unenhanced lumbar CT. Radiology 284 : 153-160

24. Rudin LI, Osher S, Fatemi E (1992) Nonlinear total variation based noise removal algorithms. Physica D 60:259-268

25. Chan TF, Shen J (2005) Image processing and analysis: variational, PDE, wavelet, and stochastic methods. Society for Industrial and Applied Mathematics (SIAM), Philadelphia

26. Zabic S, Wang Q, Morton T, Brown KM (2013) A low dose simulation tool for CT systems with energy integrating detectors. Med Phys 40:031102

27. Mei K, Kopp FK, Bippus R et al (2017) Is multidetector CT-based bone mineral density and quantitative bone microstructure assessment at the spine still feasible using ultra-low tube current and sparse sampling? Eur Radiol 27:5261-5271

28. Sollmann N, Mei K, Hedderich DM et al (2019) Multi-detector CT imaging: impact of virtual tube current reduction and sparse sampling on detection of vertebral fractures. Eur Radiol 29:3606-3616

29. Sollmann N, Mei K, Schwaiger BJ et al (2018) Effects of virtual tube current reduction and sparse sampling on MDCT-based femoral BMD measurements. Osteoporos Int 29:2685-2692

30. Sollmann N, Mei K, Schon S et al (2019) Systematic evaluation of low-dose MDCT for planning purposes of lumbosacral periradicular infiltrations. Clin Neuroradiol. https://doi.org/10. 1007/s00062-019-00844-7

31. Muenzel D, Koehler T, Brown K et al (2014) Validation of a low dose simulation technique for computed tomography images. PLoS One 9:e107843
32. Zhao Z, Gang GJ, Siewerdsen JH (2014) Noise, sampling, and the number of projections in cone-beam CT with a flat-panel detector. Med Phys 41:061909

33. Fessler JA (2000) Statistical image reconstruction methods for transmission tomography. In: Fitzpatrick JM, Sonka M (eds) Handbook of medical imaging, volume 2 medical image processing and analysis. SPIE Publications, pp 1-70

34. Kim D, Ramani S, Fessler JA (2015) Combining ordered subsets and momentum for accelerated X-ray CT image reconstruction. IEEE Trans Med Imaging 34:167-178

35. Rayudu NM, Anitha DP, Mei K et al (2020) Low-dose and sparse sampling MDCT-based femoral bone strength prediction using finite element analysis. Arch Osteoporos 15:17

36. Anitha D, Subburaj K, Kopp FK et al (2019) Effect of statistically iterative image reconstruction on vertebral bone strength prediction using bone mineral density and finite element modeling: a preliminary study. J Comput Assist Tomogr 43:61-65

37. Alshamari M, Geijer M, Norrman E et al (2017) Impact of iterative reconstruction on image quality of low-dose CT of the lumbar spine. Acta Radiol 58:702-709

38. Muckley M, Chen B, O'Donnell T et al (2018) Reconstruction of reduced-dose sparse $\mathrm{CT}$ data acquired with an interrupted-beam prototype on a clinical scanner. The Fifth international conference on image formation in X-ray computed tomography, Salt Lake City, pp 56-59

39. Chen B, Muckley M, Sodickson A et al (2018) First multislit collimator prototype for sparse CT: design, manufacturing and initial validation. The Fifth international conference on image formation in x-ray computed tomography, Salt Lake City, pp 52-55

Publisher's note Springer Nature remains neutral with regard to jurisdictional claims in published maps and institutional affiliations. 